\title{
Metallicity Studies in the IR: Unveiling Obscured Clusters of Our Galaxy
}

\author{
Francisco Najarro \\ Instituto de Estructura de la Materia, CSIC, Serrano 121, 28006 Madrid, Spain \\ email:najarro@damir.iem.csic.es
}

\begin{abstract}
We review direct and indirect methods to derive metallicity through infrared spectroscopy of massive stars. The choice of different spectral types to obtain abundances allows to trace metallicity for a wide range of ages of the cluster hosting the massive stars. These methods have a great potential to understand the evolution of large amount of heavily obscured galactic clusters, which are currently being discovered through infrared surveys.
\end{abstract}

Keywords. stars: abundances - stars: winds, outflows - stars: atmospheres - infrared: stars Galaxy: abundances

\section{Introduction}

With the advent of new detectors, the field of quantitative spectroscopy of massive stars in the infrared (IR) has undergone a major revolution during the last decade. Most of the achieved improvements in this field were triggered by the detection of a He I emission line cluster (Krabbe et al. 1991) in the central parsec of our Galaxy (GC), and our curiosity to unveil the star formation processes and energetics taking place there. The NLTE atmospheric models accounting only for H and He (Najarro et al. 1994,1997) used a decade ago to study the IR spectra of massive stars have been recently upgraded (Hillier \& Miller 1998) to account for blanketing and clumping, allowing us to obtain a detailed picture of the region (Figer et al. 2002; Najarro et al. 2004, 2006; Martins et al. 2007a, 2007b). Since the discovery of the massive cluster at the central parsec, and the Quintuplet and Arches Clusters within $30 \mathrm{pc}$ of the GC, systematic IR searches have been undertaken in the Milky Way (e.g., Dutra \& Bica 2000, Herrero et al., these proceedings). These are starting to reveal an important presence of obscured massive clusters in our Galaxy (see review by Figer, these proceedings). The presence of these clusters all over the Galaxy offers an unique opportunity to estimate metallicity and hence obtain the current two dimensional abundance pattern of our Galaxy placing, this way, crucial constraints on models of galactic chemical evolution. Interestingly, metallicity estimates of massive stars in the IR have been also triggered through observations of the three dense and massive star clusters in the GC and subsequent quantitative analysis of both their cool (Carr et al. 2000; Cunha et al. 2007) and hot (Najarro et al. 2004, 2006; Martins et al. 2007a, 2007b) stellar population. Given that the three clusters display different ages, they present an ideal laboratory to perform metallicity studies of stars at different evolutionary phases. Hence, they provide reliable templates to analyse the Galactic obscured massive clusters which are currently being discovered. In this paper, we present different methods to obtain both direct and indirect estimates metallicity of massive stars as a function of their spectral type. 

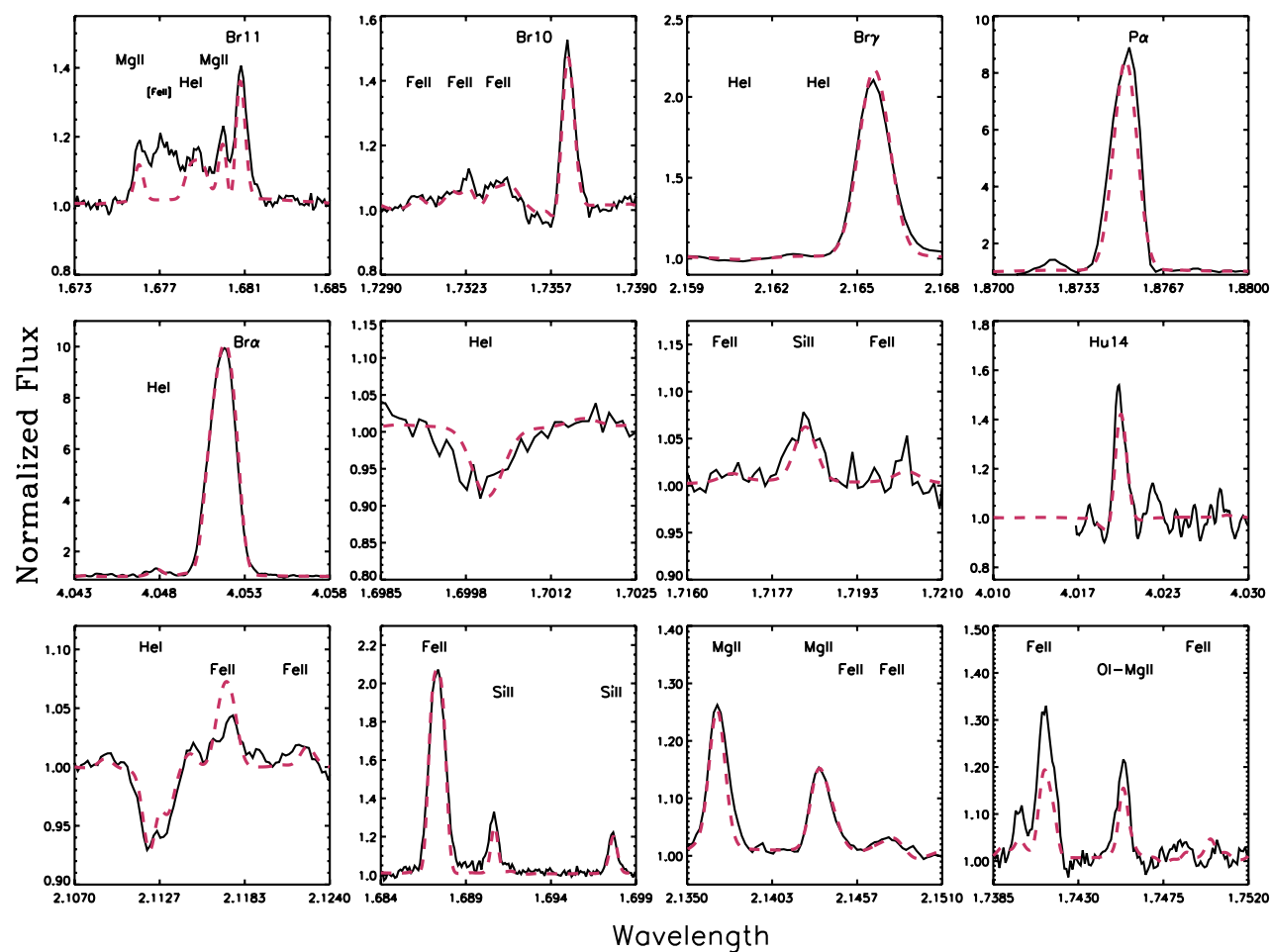

Figure 1. Model fits (dashed lines) to the observed infrared diagnostic lines (solid lines) of the Pistol Star (see Najarro et al. 2008).

\section{IR metallicity studies. What solar is solar?}

Ironically, one of the main problems arising when discussing metal abundances is which solar abundance values should be linked to solar metallicity. This controversy arises from the abundances adopted as solar in the evolutionary models, which are "delayed" with respect to the solar abundance values as determined from detailed spectroscopic modelling of the sun's photosphere. Here, we adopt the solar composition of Grevesse \& Noels (1993, hereafter GN93). Although their abundances have been recently revised Asplund et al. (2005, hereafter AS05) (but see also Pinsonneault \& Delahaye (2008)), they are the ones used by Iglesias \& Rogers(1996) to compute stellar interior opacities and adopted in the most recent evolutionary models for massive stars with rotation (Meynet \& Maeder 2003, 2005). Previously published evolutionary tracks for massive stars (Schaller et al. 1992, Meynet et al. 1994) used opacity tables calculated with solar composition from Anders \& Grevesse, (1989, hereafter AN89), which differ significantly from GN93 only in $\mathrm{Fe}(\mathrm{A}(\mathrm{Fe} / \mathrm{H})=7.67$ vs 7.50 in GN93) $\dagger$ and very slightly in the $\mathrm{CNO}$ ratios $(\mathrm{A}(\mathrm{C} / \mathrm{H})=8.56, \mathrm{~A}(\mathrm{~N} / \mathrm{H})=8.05, \mathrm{~A}(\mathrm{O} / \mathrm{H})=8.93$ in $\mathrm{AN} 89$ vs $\mathrm{A}(\mathrm{C} / \mathrm{H})=8.55$, $\mathrm{A}(\mathrm{N} / \mathrm{H})=7.97, \mathrm{~A}(\mathrm{O} / \mathrm{H})=8.87$ in $\mathrm{GN} 93)$. Si and $\mathrm{Mg}$ are the same in all evolutionary models, and have been only slightly revised downward ( $\sim 0.05 \mathrm{dex})$ by AS05. Thus, the reader should be warned about discussions of derived $\alpha$-elements vs. Fe ratio in the literature, which may depend critically on the assumed Fe solar abundance.

$$
\dagger \mathrm{A}(\mathrm{X} / \mathrm{Y})=\log [\mathrm{n}(\mathrm{X}) / \mathrm{n}(\mathrm{Y})]+12
$$




\section{Direct Methods}

Due to the lack of major features in their IR H- and K-Band spectra, hot stars are usually employed as standards when reducing IR spectra of other objects. In fact, in massive stars hot enough to allow accurate temperature determination through fitting of their HeI and HeII lines, their $\mathrm{H}$ and $\mathrm{K}$ spectra only display weak CNO features and some of them are blended. Thus, for these hotter objects, only CNO estimates may be obtained, while no direct information on $\alpha$-elements other than $\mathrm{O}(\mathrm{Si}, \mathrm{Mg}, \mathrm{Ca}$ ) or iron group elements is available. Since some of these objects will already show some N enrichment and $\mathrm{C}$ and $\mathrm{O}$ depletion from $\mathrm{C} / \mathrm{N}$ and $\mathrm{O} / \mathrm{N}$ equilibrium processes, the derived $\mathrm{CNO}$ abundances have to be interpreted with great care. For very late $\mathrm{O}$ and early $\mathrm{B}$ stars the situation is even worse as not only they run out of metal lines in their H- and K spectra but also the He II lines disappear disabling the possibility to obtain an accurate temperature determination.

However, for mid and late B stars with strong winds (LBVs and supergiants), the situation is drastically improved. Apart from the $\mathrm{H}$ and He I lines, the stars display several lines of Si II, Mg II and Fe II. Thus, a direct estimate of metallicity and $\alpha$-elements vs Fe ratio can be obtained. Note that in order to host LBVs (or late B-supergiants) the cluster has to be at least $4 \mathrm{Myr}$ old. This is case of the Quintuplet Cluster (Glas et al. 1987) which hosts two LBVs: the "Pistol Star" (Figer et al. 1998) and FMM362 (Geballe, Najarro \& Figer 2000). Najarro et al. (2008) have recently presented a detailed quantitative spectroscopic analysis of both stars and have been able to obtain direct estimates of $\mathrm{Fe}$ and $\mathrm{Mg}$ and $\mathrm{Si}$ abundances in these objects. Figure. 1 shows the excellent quality of the model fits to the relevant lines in the "Pistol Star". Below we discuss the main metal diagnostic lines (see Najarro et al. 2008 for further discussion).

Iron. Two types of Fe II lines are found in the spectra. The first are the strong semiforbidden lines that form in the outer wind and have small oscillator strengths $\left(\mathrm{gf} \sim 10^{-5}\right)$. The second are the weak permitted (gf 1) lines connecting higher lying levels that form much closer to the photosphere. The permitted lines are more robust iron abundance indicators, having only weak dependences on other parameters, such us turbulent velocity. The strengths of the semi-forbidden lines depend on the mass loss rate and the run of the iron ionization structure in the outer wind, which is sensitive to the hydrogen ionization structure due to the strong coupling to the $\mathrm{Fe} / \mathrm{H}$ charge-exchange reactions. We obtain roughly solar iron abundances for both LBVs, with \pm 0.15 dex as plausible uncertainties.

Magnesium. The strongest Mg II lines observed in the $\mathrm{H}$ and $\mathrm{K}$ bands share the $5 \mathrm{p}^{2} \mathrm{P}$ level. Those lines with it as the upper level, the $2.13 / 14 \mu \mathrm{m}$ and $2.40 / 41 \mu \mathrm{m}$ doublets (see Fig. 1) are much stronger than those with it as the lower level ( $\mathrm{H}$ band lines), revealing that pumping through the resonance $3 \mathrm{~s}^{2} \mathrm{~S}-5 \mathrm{p}^{2} \mathrm{P}$ line must be a significant populator of the $5 \mathrm{p}^{2} \mathrm{P}$ levels due to $\mathrm{Ly} \beta$ fluorescence. We estimate about twice solar $\mathrm{Mg}$ abundance and an associated uncertainty of about $\pm 0.25 \mathrm{dex}$ (due to uncertainties related to the fluorescence contribution).

Silicon. The Si II doublet $5 \mathrm{~s}^{2} \mathrm{~S}_{1 / 2}-5 \mathrm{p}^{2} \mathrm{P}_{3 / 2} 1.691 \mu \mathrm{m}$ and $5 \mathrm{~s}^{2} \mathrm{~S}_{1 / 2}-5 \mathrm{p}^{2} \mathrm{P}_{1 / 2} 1.698 \mu \mathrm{m}$ constitutes a powerful diagnostic tool, as it appears in emission for only a very narrow range of stellar temperatures and wind density structures, indicating the presence of amplified NLTE effects. However, since it forms at the base of the wind, its strong dependence on the details of the velocity field there hinders a precise silicon abundance determination. Instead, we use the well-behaved recombination line Si II $3 \mathrm{~s}^{2} 6 \mathrm{~g}^{2} \mathrm{G}$ $3 \mathrm{~s}^{2} 5 \mathrm{f}^{2} \mathrm{~F}$ at $1.718 \mu \mathrm{m}$ which shows a stronger dependence on the silicon abundance. From our model fits (see Fig. 1) we derive roughly twice solar abundance $( \pm 0.20 \mathrm{dex})$ for silicon in each LBV, similar to magnesium. 

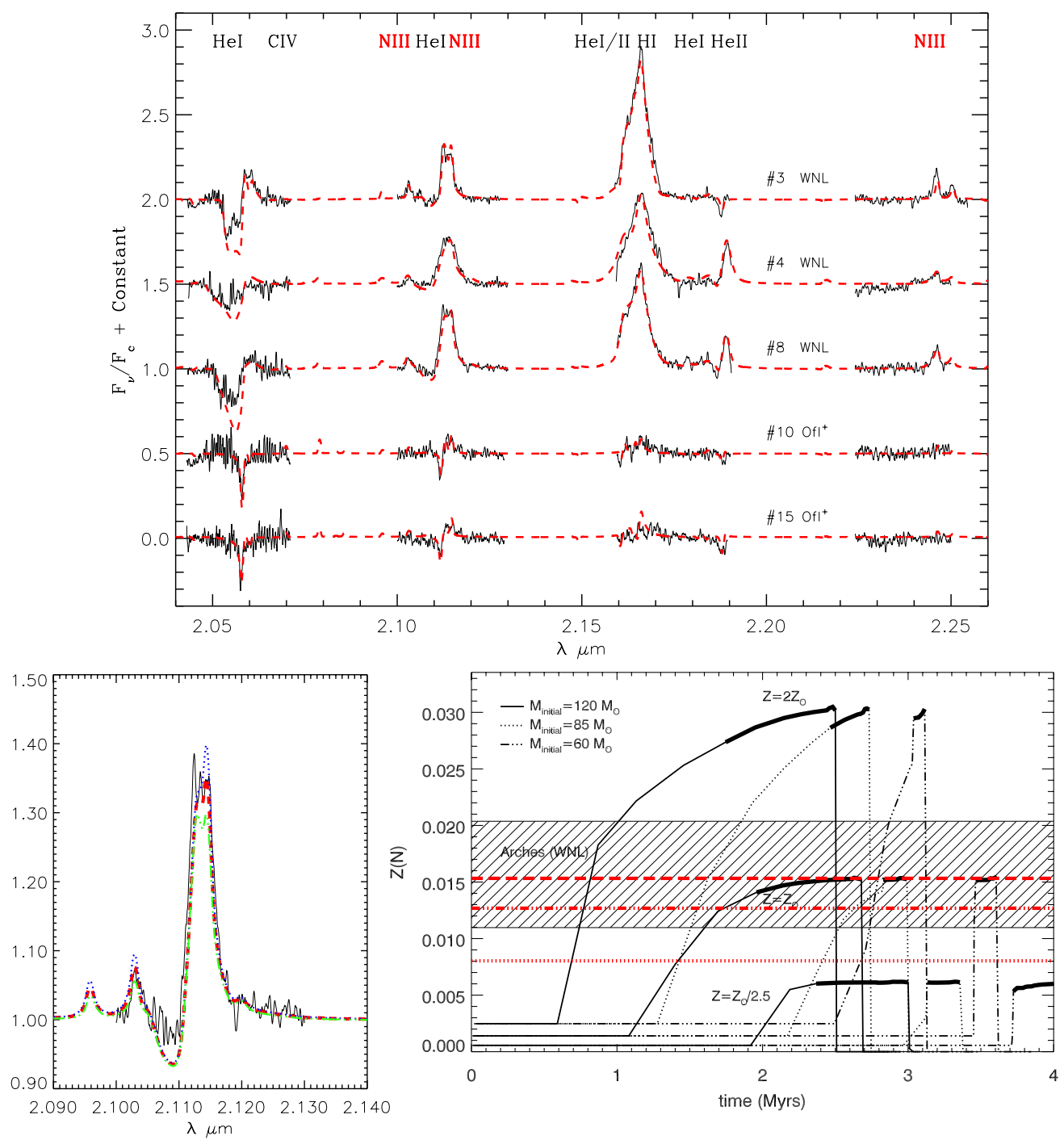

Figure 2. Top Observed spectra (solid) and model fits (dashed) for three WNL and two $\mathrm{OfI}^{+}$ Arches stars. Bottom-Left. Leverage of error estimates on $\mathrm{N}$ abundance. Best fit (dashed) and 30\% enhanced (dotted) and 30\% depleted (dashed-dotted) nitrogen mass fractions are displayed. Bottom-Right. Nitrogen mass abundance versus time using Geneva models. Maximum nitrogen surface mass fractions for solar composition are also displayed as horizontal lines for different assumed initial CNO patterns (dashed for AN89, dashed-dotted for GN93 and dotted for AS05).

\section{Indirect Methods: the WNL phase}

Najarro et al. (2004) presented a method to determine the initial oxygen abundance and, to a lesser degree, carbon abundance, of the natal cloud where the massive stars are formed. The method assumed that the star is undergoing its WNL evolutionary phase, which implies that nitrogen has reached its maximum surface abundance value while star still shows hydrogen in its spectra. Evolutionary models indicate that $95 \%$ of that value is already attained by the time that $\mathrm{H} / \mathrm{He}<2$ (by number). Such scenario is currently met by the most massive stars in the Arches cluster and some WN stars in the Central parsec 


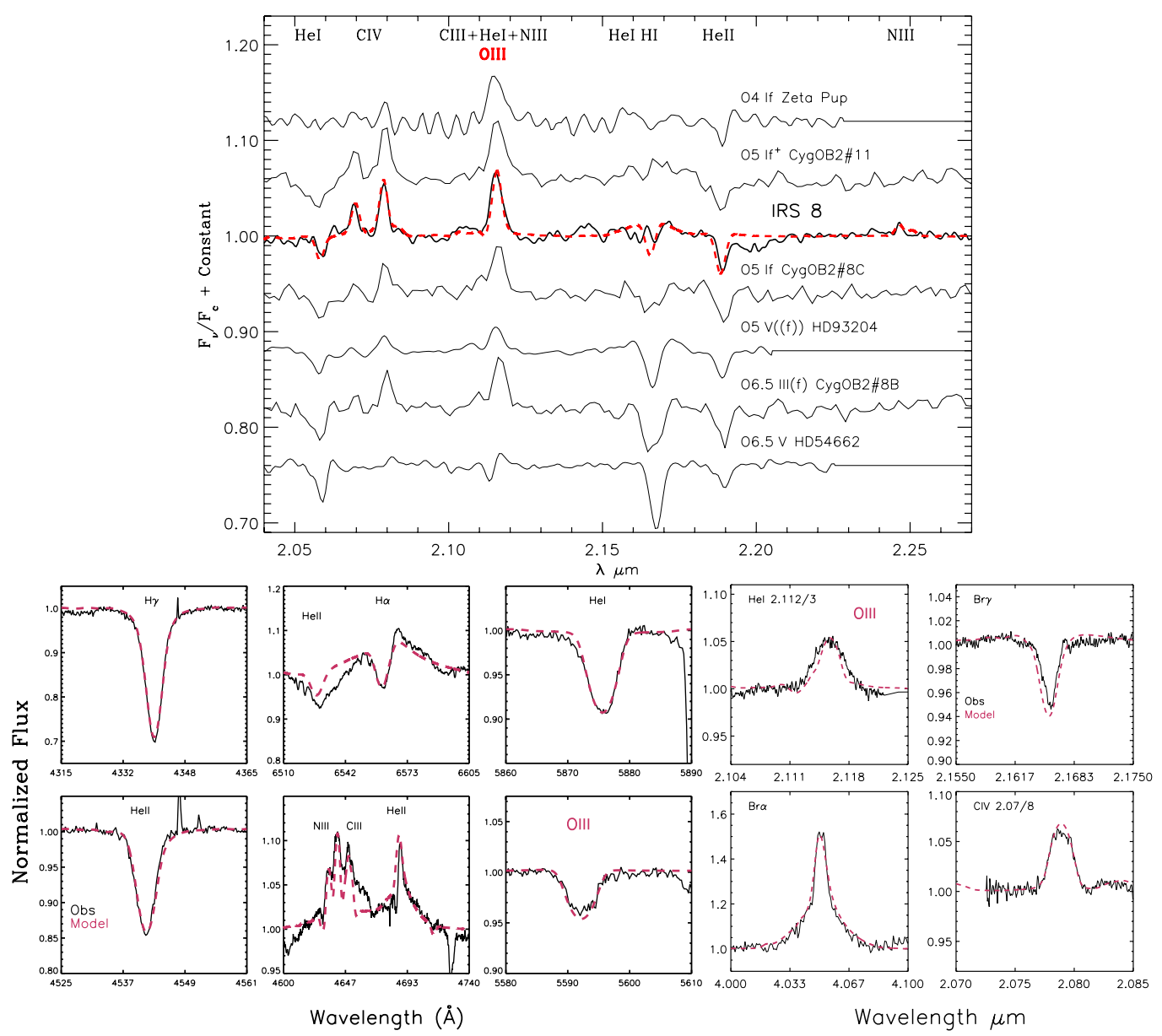

Figure 3. Top Spectral type determination of IRS $8^{*}$ and identification of the O III $2.116 \mu \mathrm{m}$ feature. Model fit (dashed) with stellar parameters corresponding to an O5.5If star. Bottom Consistency check of the method through simultaneous fitting of the optical and IR lines, including oxygen, for CygOB2 \#8C. (Najarro et al. in prep.)

cluster. Being very young $(\leqslant 2.5 \mathrm{Myr})$, the Arches cluster hosts WNLs and $\mathrm{OIf}^{+}$having infrared spectra dominated by H, He I, He II, N III lines. Some have weak C III/IV lines (see Fig. 2). Figer, McLean, \& Najarro(1997) showed that these N III lines appear only for a narrow range of temperatures and wind densities, which occur in the WN9h (WNL) stage. The fairly distinct nature and energies of the multiplets involved in each of both N III line sets provide strong constraints for the determination of the nitrogen abundance. Najarro et al. (2004) followed the metallicity patterns from the Geneva evolutionary models and assumed no selective enrichment of CNO or $\alpha$-elements vs Fe, in concluding that the stars in the Arches Cluster have solar $\alpha$-element abundances (see Fig. 2). However, estimates of solar abundances have varied considerably over the past 15 years. Thus, depending on the assumed solar CNO composition, the derived nitrogen abundance by Najarro et al. (2004) could imply solar (AN89), $1.2 \times$ solar (GN93) or $2.0 \times$ solar (AS05) CNO composition (see Fig. 2). Using the same approach, Martins et al. (2007a, 2007b) have recently analyzed a larger sample of hot stars in the Arches and Central Parsec clusters and find similar results. Interestingly, if one considers only the objects in Martins et al. 
(2008) with $\mathrm{He} / \mathrm{H}>0.1$ and those with $\mathrm{Z}(\mathrm{C})<0.05$, i.e., fulfilling the condition to be close enough to $\mathrm{Z}(\mathrm{N})_{\max }$, their average value of $\mathrm{Z}(\mathrm{N})$ shows excellent agreement with the one obtained by Najarro et al. (2004).

\section{New direct method: Oxygen abundance in early and mid $\mathrm{O}$ stars}

Again, the GC has played a starring role, as the identification of the central source of IRS 8 in the cluster at the central parsec as a mid O supergiant (Geballe et al. 2006) enabled the development of a new method to estimate metallicity in these objects. When addressing the spectral type classification of IRS $8^{*}$ by comparing its K-band spectrum with on line-available K-band spectra (Hanson et al. 1996, see Fig.3, top), Geballe et al. (2006) raised the question about the nature of the emission feature at $2.116 \mu \mathrm{m}$. This had been attributed in the past to $\mathrm{C}$ III and $\mathrm{N}$ III $\mathrm{n}=8-7$ transitions and is present over a very wide range of $\mathrm{O}$ spectral types and luminosities (Hanson et al. 1996). Our investigation (Geballe et al. 2006) indicated that the $2.116 \mu \mathrm{m}$ feature in IRS $8^{*}$ is dominated by O III $n=8-7$ transitions. Further, the O III component of the $2.116 \mu \mathrm{m}$ feature largely depends on the oxygen abundance and only slightly on gravity, effective temperature, wind density, and velocity field. Hence, this feature constitutes a powerful diagnostic of oxygen abundance, and therefore an important metal abundance determiner, over a wide range of $\mathrm{O}$ spectral types (Najarro et al. in preparation). Given the strong spectral similarities of IRS 8* with the O5-6 supergiants in Cyg OB2 (see Fig. 3), we calibrated our method and checked for consistency by fitting simultaneously our available UV, optical and IR spectra of stars in Cyg OB2. Figure 3-bottom demonstrates the consistency of our fits to the optical and IR spectra for Cyg OB2\#8C (O5If), and the excellent agreement of the models with both $\mathrm{O}$ III lines. The oxygen abundance of $\mathrm{A}(\mathrm{O} / \mathrm{H})=8.91$ obtained IRS $8^{*}$ is fully consistent with the values obtained for the Arches WNLs and the $\alpha$ element enrichment derived for the Quintuplet LBVs, providing a consistent picture of the metallicity in the GC.

\section{Acknowledgement}

I would like to thank Don Figer, John Hillier, Rolf Kudritzki and Tom Geballe for invaluable discussions. F. N. acknowledges AYA2004-08271-C02-02 grant. Special thanks to Miguel \& Fabio for the great late sessions organized at Stevenson's Library.

\section{References}

Anders, E. \& Grevesse, N., 1989, Geochimica et Cosmochimica Acta, Vol. 53, 197

Asplund, ., Grevesse, N., \& Sauval, A. J. 2005, in: T. G. Barnes III \& F. N. Bash (eds.), Cosmic Abundances as Records of Stellar Evolution and Nucleosynthesis (San Francisco: ASP), ASP Conf Ser, 336, 25

Dutra, C. M. \& Bica, E., 2000, A\&A, 359, 9

Carr, J. S., Sellgren, K. \& Balachandran, S. C. 2000, ApJ, 530, 307

Cunha, K., Sellgren, K., Smith, V. V. et al. 2007, ApJ, 669, 1011

Figer, D. F., Najarro, F., Morris, M., et al. 1998, ApJ, 506, 384

Figer, D. F., McLean, I. S., \& Najarro, F. 1997, ApJ, 486, 420

Figer, D. F., Najarro, F., Gilmore, D. et al. 2002, ApJ, 581, 258

Geballe, T. R., Najarro, F., \& Figer, D. F. 2000, ApJ, 530, L97

Geballe, T. R., Najarro, F., Rigaut, F., \& Roy, J.-R. 2006, ApJ, 652, 370

Glass, I. S., Catchpole, R. M., \& Whitelock, P. A. 1987, MNRAS, 227, 373

Grevesse, N. \& Noels, A. 1993, N. Prantzos, E. Vangioni-Flam \& M. Casse (eds.), Origin and evolution of the elements (Cambridge: CUP), 14 
Hanson, M. M., Conti, P. S., \& Rieke, M. J. 1996, ApJS, 107, 281

Hillier, D. J. \& Miller, D. L. 1998, ApJ, 496, 407

Iglesias, C. A. \& Rogers, F. J. 1996, ApJ, 464, 943

Krabbe, A., Genzel, R., Drapatz, S., \& Rotaciuc, V., 1991, ApJ, 382, L19

Martins, F., Genzel, R., Hillier, D. J. et al. 2007, A\&SA, 468, 233

Martins, F., Hillier, D. J., Paumard, T. et al., 2008, A\& $A$, 478, 219

Meynet, G., Maeder, A., Schaller, G., Schaerer, D., \& Charbonnel, C. 1994, A\&AS, 103, 97

Meynet, G. \& Maeder, A. 2003, A\&3A, 404, 975

Meynet, G. \& Maeder, A. 2005, A\&BA, 429, 581

Moneti, A., Glass, I. S. \& Moorwood, A. F. M. 1994, MNRAS, 268, 194

Nagata, T., Woodward, C. E., Shure, M. et al. 1990, ApJ, 351, 83

Najarro, F., Hillier, D. J., Kudritzki, R.-P. et al. 1994, A\& A, 285, 573

Najarro, F., Krabbe, A., Genzel, R. et al. 1997, A\&A, 325, 700

Najarro, F., Hillier, D. J., Figer, D. F., \& Geballe, T. R. 1999, in: H. Falcke, A. Cotera, W. J. Duschl et al. (eds.), The Central Parsecs of the Galaxy, (San Francisco: ASP), ASP Conf Ser, 186,340

Najarro, F. 2001, in: M. de Groot \& C. Sterken (eds.), P Cygni 2000: 400 Years of Progress, (San Francisco: ASP), ASP Conf Ser, 233, 133

Najarro, F., Figer, D. F., Hillier, D. J., \& Kudritzki, R. P. 2004, ApJ, 611, L105

Najarro, F. 2006, Journal of Physics: Conf. Ser., 54, 224

Najarro, F., Figer, D. F., Hillier, D. J., Geballe, T. R., \& Kudritzki, R. P. 2008, ApJ, submitted Pinsonneault, M. H., \& Delahaye, F. 2008, ApJ, submitted (arXiv:astro-ph/0606077)

Schaller, G., Schaerer, D., Meynet, G., \& Maeder, A. 1992, A\&AS, 96, 269

\section{Discussion}

STOLOVY: Why, of all of the massive stars that you have discussed, is the Pistol Star the only one with a nebula around it? Why doesn't the LBV candidate FMM362 have a nebula?

NAJARRO: It could very well be, as in the case of P Cygni, that for FMM362 the nebula may be there but very faint to be detected.

LEITHERER: What do you know about the abundances in nearby giants and supergiants? The comparison can give insight into the chemical evolution and also confidence in the modeling.

NAJARRO: As I showed, we are currently investigating these stars in Galactic regions, like the Cyg OB2 cluster where observations are available from the UV to the optical. First results seem to confirm consistency between optical and IR studies. 


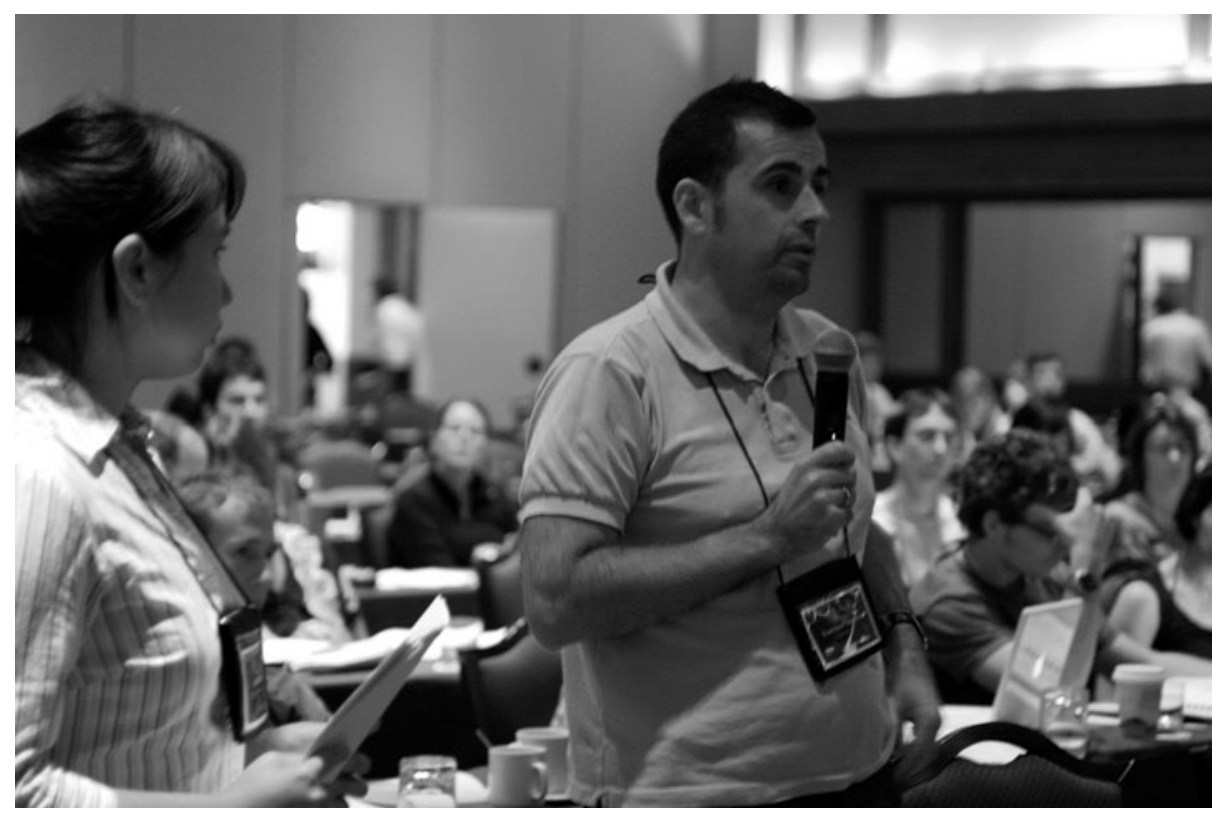

Paco Najarro.

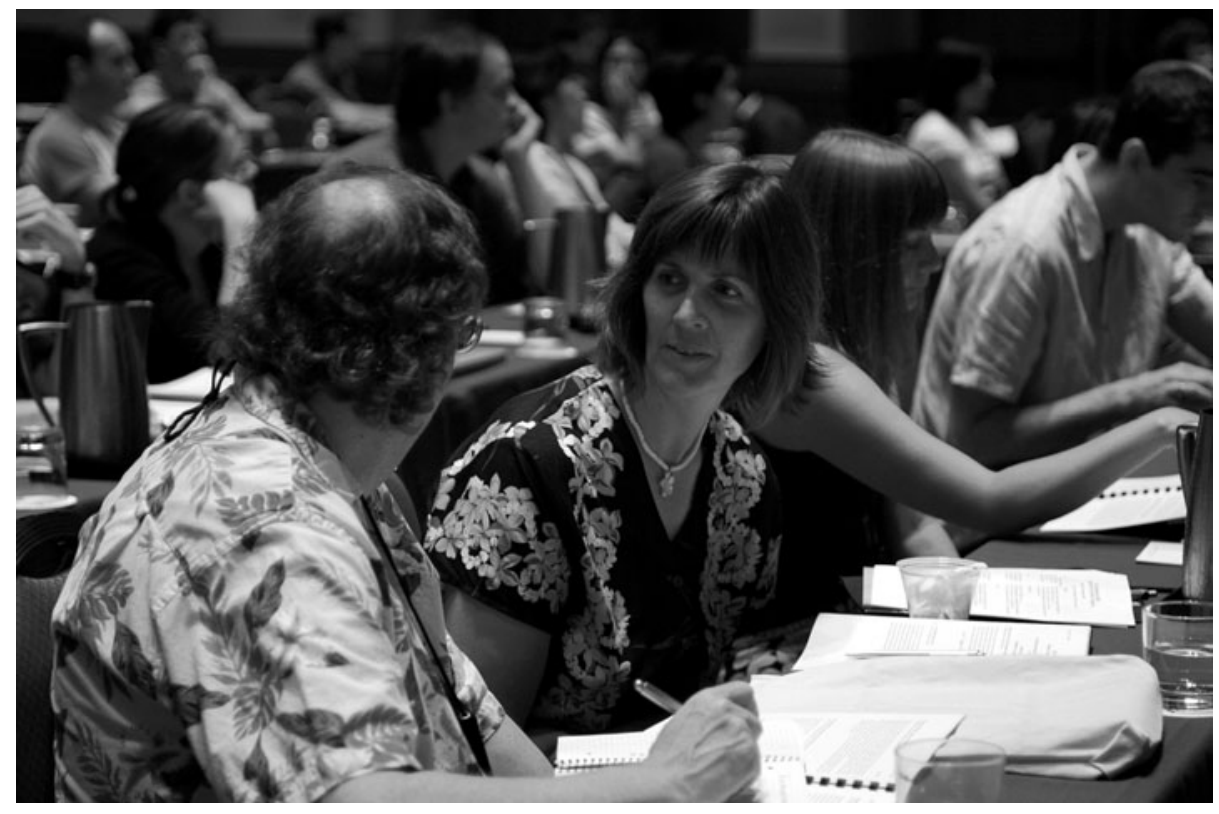

Margaret Hanson. 